\title{
Natural and non-natural antioxidative compounds: potential candidates for treatment of vascular calcification
}

Chia-Ter Chao 1,2,3, Hsiang-Yuan Yeh ${ }^{4}$, You-Tien Tsai ${ }^{3}$, Pei-Huan Chuang ${ }^{3}$, Tzu-Hang Yuan ${ }^{3}$, Jenq-Wen Huang ${ }^{5}$ and Huei-Wen $\mathrm{Chen}^{3}$

\begin{abstract}
Vascular calcification (VC) is highly prevalent in patients with advanced age, or those with chronic kidney disease and diabetes, accounting for substantial global cardiovascular burden. The pathophysiology of $\mathrm{VC}$ involves active mineral deposition by transdifferentiated vascular smooth muscle cells exhibiting osteoblast-like behavior, building upon cores with or without apoptotic bodies. Oxidative stress drives the progression of the cellular phenotypic switch and calcium deposition in the vascular wall. In this review, we discuss potential compounds that shield these cells from the detrimental influences of reactive oxygen species as promising treatment options for VC. A comprehensive summary of the current literature regarding antioxidants for $\mathrm{VC}$ is important, as no effective therapy is currently available for this disease. We systematically searched through the existing literature to identify original articles investigating traditional antioxidants and novel compounds with antioxidant properties with regard to their effectiveness against VC in experimental or clinical settings. We uncovered 36 compounds with antioxidant properties against VC pathology, involving mechanisms such as suppression of NADPH oxidase, BMP-2, and Wnt/B-catenin; anti-inflammation; and activation of Nrf2 pathways. Only two compounds have been tested clinically. These findings suggest that a considerable opportunity exists to harness these antioxidants for therapeutic use for VC. In order to achieve this goal, more translational studies are needed.
\end{abstract}

\section{Facts}

1. Anti-oxidants are compounds that counteract oxidative stress within cellular microenvironments, divided broadly into those of natural and nonnatural origin.

2. Reactive oxygen species are vital pathogenic players in atherosclerotic cardiovascular diseases, against which anti-oxidants can be excellent sources of treatment candidates.

Correspondence: Huei-Wen Chen (shwchen@ntu.edu.tw)

'Department of Medicine, National Taiwan University Hospital BeiHu Branch, College of Medicine, National Taiwan University, Taipei, Taiwan

${ }^{2}$ Department of Internal Medicine, National Taiwan University College of

Medicine, Taipei, Taiwan

Full list of author information is available at the end of the article.

Edited by I. D'agnano
3. Vascular calcification (VC) contributes significantly to cardiovascular morbidity and mortality in at-risk population including those with diabetes and kidney diseases, but effective therapies for $\mathrm{VC}$ remain in question.

\section{Open questions}

1. To what extent anti-oxidative therapies have been utilized to treat VC experimentally and clinically?

2. What molecular mechanisms are involved in the observed therapeutic efficacy of antioxidants against VC?

3. How to choose the optimal dose and duration of different anti-oxidants for the treatment of $\mathrm{VC}$ in light of the existing literature?

\section{(c) The Author(s) 2019}

(c) (i) Open Access This article is licensed under a Creative Commons Attribution 4.0 International License, which permits use, sharing, adaptation, distribution and reproduction c. in any medium or format, as long as you give appropriate credit to the original author(s) and the source, provide a link to the Creative Commons license, and indicate if changes were made. The images or other third party material in this article are included in the article's Creative Commons license, unless indicated otherwise in a credit line to the material. If material is not included in the article's Creative Commons license and your intended use is not permitted by statutory regulation or exceeds the permitted use, you will need to obtain permission directly from the copyright holder. To view a copy of this license, visit http://creativecommons.org/licenses/by/4.0/. 


\section{Introduction}

Cardiovascular diseases are the leading cause of mortality worldwide, accounting for nearly one-fourth of total deaths globally, while real-world data suggest that the burden is likely higher than we deduce based on trial information ${ }^{1}$. Despite the substantial benefit conferred by the widespread use of statin for cholesterol reduction as well as the promotion of guideline adherence, the global mortality due to cardiovascular diseases continued to increase from the 1990s to 2013 (ref. ${ }^{2}$ ). This trend has been partly attributed to population aging, but risk factors other than demographic influences likely contribute as well, such as atypical factors including chronic inflammation, mineral dysregulation, and excess pathogenic hormonal milieu ${ }^{3}$. Findings from previous studies suggested vascular calcification (VC) as an important phenotype arising from the influences of atypical cardiovascular risk factors ${ }^{4}$. Indeed, $\mathrm{VC}$ is highly prevalent in certain patient populations, including those with chronic kidney disease (CKD) or diabetes mellitus, and the presence of $\mathrm{VC}$ is predictive of an increased risk of cardiovascular diseases, overall mortality, and hospitalization independent of multiple confounders ${ }^{5}$. This detrimental influence of $\mathrm{VC}$ is mainly related to enhanced arterial stiffness, ventricular pressure load with hypertrophy and coronary ischemia. However, effective therapies for VC remain scant, and even the promising ones involve the optimization of divalent ions and interplay between hormones within the scope of mineral bone disorder without proven efficacy ${ }^{6}$. Consequently, novel therapies that tackle alternative aspects of VC pathophysiology are urgently needed.

\section{The central role of oxidative stress in VC}

The pathophysiology of $\mathrm{VC}$ is complex, but most agree that active osteoid matrix-like deposition by transdifferentiated vascular smooth muscle cells (VSMCs) or infrequently by other vascular wall constituent cells plays an important role. This highly regulated cellular phenotypic switch, accompanied by the loss of contractile function (for VSMCs) and the gain of synthetic function, can be stimulated by disturbed mineral homeostasis (especially a high phosphate environment), hyperglycemia with advanced glycation endproduct (AGE) accumulation, or inflammatory cytokines, which coincide with the clinical features of the high-risk population for developing $\mathrm{VC}^{7}$. The process of active mineralization is further augmented by the loss of calcification inhibitors such as matrix Gla protein (MGP), pyrophosphate, or fetuin- $\mathrm{A}^{8}$.

A core component that drives the phenotypic change of VSMCs during VC is believed to be premature vascular aging and VSMC senescence ${ }^{9}$. Oxidative stress (OS), manifested by the production of reactive oxygen species (ROS) and counteracted by antioxidant mechanisms, is a major inducer of cellular senescence. OS presumably induces damages to vital cellular substructures including DNA, protein, and membrane/cytoplasmic lipids. The sources of ROS in calcified VSMCs include cellular enzymes such as xanthine oxidase, nicotinamide adenine dinucleotide phosphate oxidase (NADPH oxidase, Nox), and the cytochrome P450 systems. The downstream events of redox imbalance may include telomere shortening, mitochondrial DNA damage, and direct mutagenic influences on gene fragments involved in contractile functions, thereby predisposing VSMCs to aging and the loss of smooth muscle cell phenotypes. These events are followed by osteogenic transcription factor activation ${ }^{10,11}$. In addition, mitochondrial damage can be accompanied by further leakage of ROS from inner mitochondrial membrane, perpetuating a vicious cycle of ROS accumulation and a greater degree of apoptosis. Inflammation following cytokine release triggered by OS can further set the stage for $\mathrm{VC}$ development.

In light of the importance of ROS in the pathogenesis of $\mathrm{VC}$, it is expected that antioxidants can serve as a promising therapeutic approach for managing $\mathrm{VC}$, a disorder currently without effective treatments ${ }^{12}$. We thus performed a literature-based review to summarize the existing knowledge with regard to the utility of antioxidants against $\mathrm{VC}$, in experimental models and in clinical studies.

\section{Strategy of literature search}

We systematically searched through the existing literature, using the following combinations of keywords and MESH terms: "vascular smooth muscle cells", "calcification", or "vascular calcification" and "reactive oxygen species", "oxidative stress", "antioxidant", or "anti-oxidant" in the PUBMED and MEDLINE databases up to 7 June 2019 to retrieve relevant articles for analysis; 310 articles were identified, of which 101 were excluded as duplicate entries (Fig. 1). We subsequently reviewed the abstracts of the remaining 209 articles in depth, and excluded review articles $(n=53)$ and those for which the full text was unavailable $(n=1)$, after which these articles were classified into clinical $(n=47)$ and experimental $(n=108)$ research studies. For clinically oriented studies, we further excluded those whose focuses were not therapeutic, therapeutic but not against VC, or case reports; for experimental ones, we excluded those not examining chemicals, examining pathogenesis only, examining non-VC pathologies, or those examining aggravating factors (Fig. 1).

The remaining 52 articles with their full-texts were finally reviewed with data extracted for description. Among these, 5 (9.6\%) involved clinical studies that examined antioxidants for treatment of $\mathrm{VC}$, while 47 (90.4\%) involved experimental studies. For clinical therapeutic studies, four (80\%) used parenteral sodium 


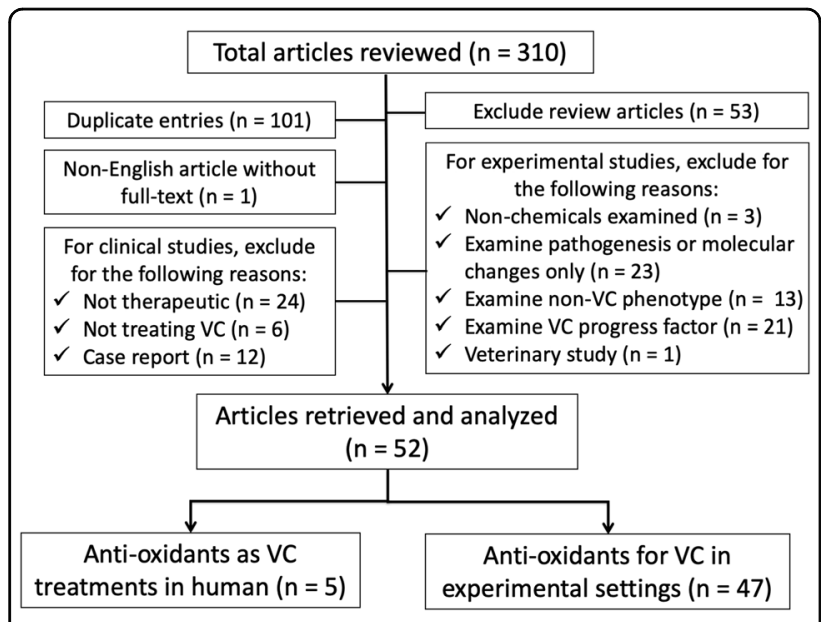

Fig. 1 The algorithm of study retrieval from the literature and the application of selection criteria. VC vascular calcification

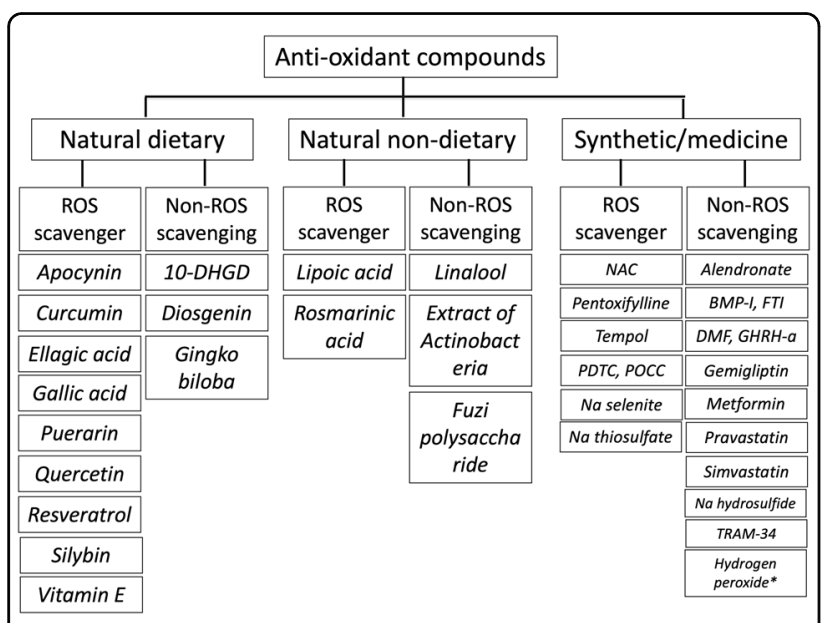

Fig. 2 The proportion of identified antioxidant compounds within each category, stratified based on the presence or absence of ROS scavenging ability. 10-DHGD 10-

dehydrogingerdione, BMP-I bone morphogenetic protein inhibitor, DMF dimethylfumarate, FTI farnesyl transferase inhibitor, GHRH-a growth hormone-releasing hormone receptor agonist, NAC Nacetylcysteine, PDTC pyrrolidine dithiocarbamate, POCC poly(1,8octamethylene-citrate-co-cysteine), ROS reactive oxygen species. * Very low dose $(0.01 \mathrm{mM})$

thiosulfate (STS), while one (20\%) used topical cerium nitrate $^{13-17}$. For experimental approaches, totally 36 compounds with antioxidant property have been tested against $\mathrm{VC}$, including 12 natural dietary substances, 5 natural non-dietary substances, and 19 pharmaceuticals or synthetic chemicals (Fig. 2) ${ }^{18-64}$. Among compounds from each category, they are further divided into those with and without ROS scavenging ability.
We examined candidate antioxidants with regard to their effects on $\mathrm{VC}$ and the potential mechanisms involved if the articles used experimental approaches, and the antioxidants that have been tested for therapeutic efficacy against $\mathrm{VC}$ in clinical settings, in the following sections. Compounds which have been tested in animal models are tabulated separately to outline the dosage and models through which anti-calcification effect are shown separately (Table 1 ).

\section{Antioxidants from natural and dietary sources for treatment of VC}

To date, in vitro and in vivo experiments have tested 12 natural antioxidants that are also dietary ingredients, including 10-dehydrogingerdione (10-DHGD $)^{64}$, apocynin $^{18,19}$, curcumin $^{38}$, diosgenin ${ }^{20,21}$, ellagic acid ${ }^{56}$, gallic acid $^{48}$, gingko biloba extracts ${ }^{50}$, puerarin ${ }^{62}$, quercetin ${ }^{25-31}$, resveratrol ${ }^{52}$, silybin ${ }^{38}$, and vitamin $\mathrm{E}^{34,35}$. Among these compounds, apocynin, diosgenin, quercetin, and vitamin $\mathrm{E}$ have been shown by multiple studies to have anti-VC properties. Nine (75\%) of these compounds possess ROS scavenging ability, while others do not (Fig. 2).

Apocynin is isolated from the plant Apocynum, also known as dogbane, and has been used as a herbal medicine by Native Americans and as herbal tea in several Asian countries ${ }^{65}$. Through inhibition of Nox, apocynin has been found to reduce superoxide generation and ameliorate inflammation in various animal models ${ }^{66}$. Brodeur et al. found that the administration of apocynin to streptozotocin-induced diabetic rats with warfarininduced arterial calcification could substantially improve the severity of femoral artery $\mathrm{VC}$, although the mechanism involved has not been tested ${ }^{18}$. Other studies further disclosed that apocynin ameliorated VC by suppressing bone morphogenetic protein-2 (BMP-2) expression and attenuating the phosphorylation of ERK1/2, thereby reducing RUNX2 and osteopontin levels based on an angiotensin II-induced osteogenic transformed VSMCs model $^{19}$. Diosgenin is a phytosteroidal saponin purified from fenugreek (Trigonella foenum-graecum) seeds and can be biosynthesized from cholesterol. Previous studies demonstrated that diosgenin possessed lipid and glucoselowering effects in addition to anti-inflammatory and antioxidant properties ${ }^{67}$. Manivannan et al. found that diosgenin could reduce aortic calcification in CKD rats in a dose-dependent manner by increasing antioxidant enzyme levels, lowering lipid peroxidation ${ }^{20}$, and stimulating endothelial nitric oxide synthase (eNOS) activities, thereby improving coronary reserves ${ }^{21}$. Vitamin E, including lipophilic tocopherols and tocotrienols, is universally found in green vegetables, nuts, eggs, and milk, and has long been known to exhibit strong antioxidant properties. Mody et al., in a pilot study nearly two decades ago, found that a vitamin $\mathrm{E}$ analog could halt the 


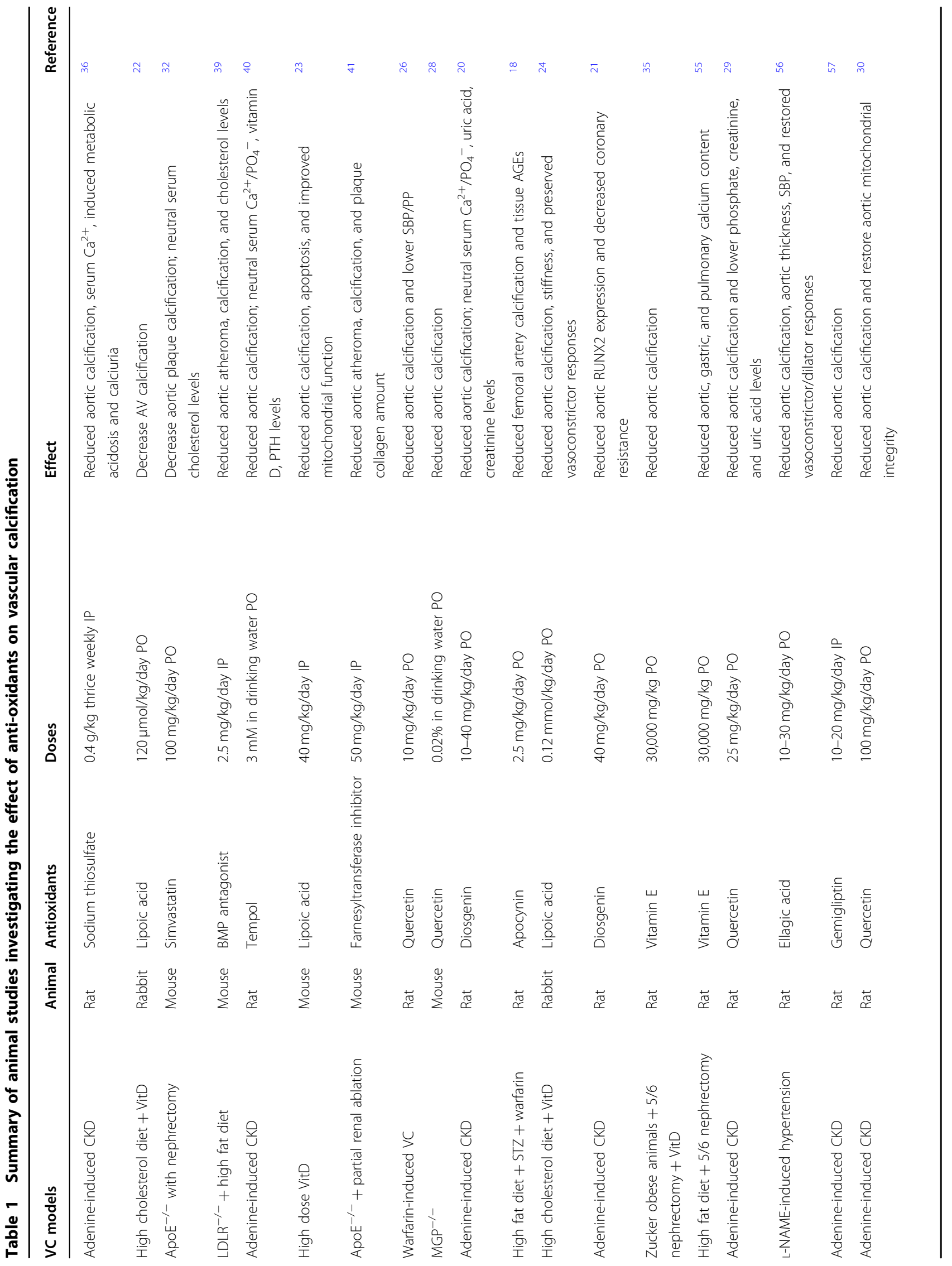




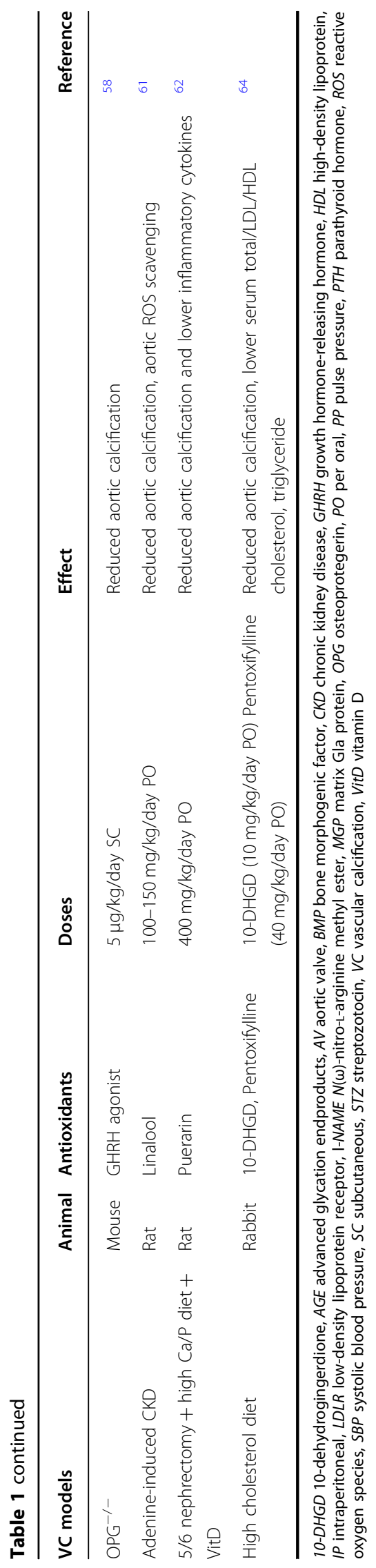

osteoblastic differentiation of aortic VSMCs induced by oxidized low-density lipoprotein $(\mathrm{ox}-\mathrm{LDL})^{34}$. Vitamin E has subsequently been shown to restore plasma antioxidant levels, reduce AGEs, and suppress aortic calcification severity in obese CKD rats and in human VSMCs ${ }^{35}$.

Quercetin, a naturally occurring flavanol and a secondary plant metabolite, is enriched in many fruits and vegetables and is also used as a dietary supplement ${ }^{68}$. It has been extensively investigated with respect to its effect against VC based on our literature search results. Lu et al. disclosed that quercetin abolished heat shock protein 72 induced anti-calcification effect in vitro and ex vivo ${ }^{25}$; however, another group later found that quercetin completely nullified warfarin-induced aortic calcification and aortic medial cartilaginous metaplasia by inhibiting transglutaminase 2 and $\beta$-catenin expression in vitro ${ }^{26-28}$. Alternatively, quercetin could reduce aortic calcification in CKD rats partially by increasing superoxide dismutase 2 (SOD2) levels and modulating the inducible NO synthase (iNOS)/MAPK pathway ${ }^{29}$. Cui et al. further showed that quercetin restored mitochondrial integrity with improved membrane potential, ATP production, reduced fragmentation/fission, attenuated Drp1 phosphorylation, and reversed the apoptotic process in calcified VSMCs ${ }^{30}$. Recently, Liang et al. showed that quercetin might be protective for VSMCs by attenuating the expression of BMP-2 and toll-like receptor (TLR)-4 in ox-LDLinduced calcification ${ }^{31}$.

On the other hand, the antioxidant properties of 10DHGD, curcumin, ellagic acid, gallic acid, gingko biloba, puerarin, resveratrol, and silybin were described in single reports only. 10-DHGD is a biologically active component extracted from the rhizomes of ginger (Zingiber officinale), a medicinal plant that is also used as dietary spices $^{69}$. 10-DHGD has been shown to exhibit antiinflammatory and antioxidant properties, and to stimulate NO release ${ }^{70}$. Elseweidy et al. revealed that 10-DHGD could attenuate the development of aortic atherosclerotic calcifications in dyslipidemic rabbits by downregulating the expression of aortic BMP-2, Wnt3a, tumor necrosis factor- $\alpha$ (TNF- $\alpha$ ), receptor activator of nuclear factor $-\mathrm{kB}$ (RANK), and RUNX2 ${ }^{64}$. Curcumin is a polyphenol extracted from the dried Rhizomes of Curcuma longa and spices such as turmeric. It is known to possess antiinflammatory and antioxidant properties ${ }^{71}$. Silybin is the main active ingredient of Silybum marianum extracts. It exhibits antioxidant property and is mostly used for treating viral hepatitis ${ }^{72}$. Roman-Garcia et al. showed that curcumin and silybin reduced ROS levels and calcification of VSMCs ${ }^{38}$. Being a natural polyphenol with antioxidant property, gallic acid and its derivative, ellagic acid, mainly exist in fruits such as pomegranates, grapes, berries, and tea leaves ${ }^{73}$. Gallic acid has been shown to reduce osteoblastic differentiation of VSMCs by suppressing 
BMP-2/Smad 1/5/8 signaling, while ellagic acid has been shown to decrease calcium deposition in aortic walls and improves blood pressure in hypertensive rats ${ }^{48,56}$. Ginkgo biloba extracts are mixtures containing several flavanols, ginkgolides, and bilobalides, and are found to be vasoactive and antioxidative ${ }^{74}$. Li et al. demonstrated that Ginkgo biloba could attenuate VSMC calcification by downregulating NF- $\mathrm{kB}$ and reducing ROS levels ${ }^{50}$. The primary extract of Pueraria lobata, puerarin, is used as an herbal medicine for treating acute ischemic stroke. Its active ingredient is an isoflavone glycoside ${ }^{75}$. Liu et al. disclosed that puerarin inhibited ROS production, reduced NF- $\mathrm{kB}$ and BMP-2 expression, and IL-1 $\beta$ levels, resulting in reduced aortic calcification ${ }^{62}$. Finally, resveratrol, a phytoalexin and polyphenol mainly contained in plants such as grapes and its derivatives such as red wine, has been repeatedly shown to be vasculo- and cardio-protective ${ }^{76}$. Zhang et al. found that resveratrol reduced VSMC calcification by activating Nrf2 and Sirt1, upregulating Klotho expression and inhibiting fibroblast growth factor-23 (ref. ${ }^{52}$ ).

Based on the above reports, there is a high probability that multiple edible natural antioxidants can be potential candidates for managing $\mathrm{VC}$, although the doses of each agent vary widely, and the mechanisms may differ depending on agent-specific or experimental modelspecific issues. We have constructed an illustrative diagram to integrate the known and confirmed molecular alterations responsible for the beneficial effects of these nutraceuticals (Fig. 3).

\section{Antioxidants from natural non-dietary sources}

We identified five types of potential antioxidants with therapeutic effects against VC based on the literature search, including linalool ${ }^{61}$, lipoic acid ${ }^{22-24}$, rosmarinic acid $^{63}$, fermentation broth extracts from Actinobacteria ${ }^{59}$, and polysaccharide from Fuzi product ${ }^{60}$. Two $(40 \%)$ of them have ROS scavenging ability, while the other three (60\%) do not (Fig. 2).

Linalool, a natural terpene alcohol inherent to multiple types of flowers, is now a common constituent of fragrance with cosmetic and non-cosmetic applications ${ }^{77}$. Kaur et al. disclosed that linalool could scavenge ROS in VSMCs, increase antioxidant enzyme levels, and reduce the expression of PiT-1 and RUNX2 in CKD rats ${ }^{61}$. Lipoic acid, produced from octanoic acid in mitochondria, is a cofactor for multiple physiological reactions and a powerful antioxidant known to date. Several groups have proposed that lipoic acid could substantially attenuate VC in vitro and in vivo. Liberman et al. showed that lipoic acid ameliorated aortic calcification and improved vascular compliance in high cholesterol- and vitamin D-fed rabbits by inhibiting Nox4 expression and reducing $\operatorname{ROS}^{22,24}$. Similar to quercetin, lipoic acid exhibited a beneficial effect against VC by improving mitochondrial function and inhibiting apoptosis, through restoring Gas6/Axl survival signaling ${ }^{23}$. Rosmarinic acid is a phenolic compound purified from medicinal plants belonging to the families Apiaceae, Boraginaceae, and Lamiaceae with anti-inflammatory and antioxidant properties ${ }^{78}$. Ji et al. showed that rosmarinic acid reduced VSMC calcification by upregulating Nrf2, NADPH quinone dehydrogenase 1 (NQO1) expression, increasing antioxidant enzyme levels while inhibiting NF- $\mathrm{KB}$ and $\beta$-catenin signaling in vitro and in vivo ${ }^{63}$. Finally, extracts of bacterial and plant products are also found to counteract VC experimentally. Preliminary results from Liao et al. showed that polysaccharide extracted from Radix Aconiti Carmichaeli, a raw product with anti-oxidant activity, could reduce calcification severity of ox-LDL-treated VSMCs, partially by activating autophagy ${ }^{60}$. Salimi et al. also found that metabolites extracted from cultured Nocardia strain could attenuate protein oxidation, reduce inflammation, and reduce VSMC calcification severity ${ }^{59}$. The potential downstream effectors of these natural nondietary compounds are summarized in Fig. 3. Although parts of the above compounds are derived by extracting botanical and bacterial metabolites and are likely heterogeneous, these findings suggest that a plentiful of natural substances can be tested as powerful treatments for VC.

\section{Antioxidants of synthetic origin or pharmaceuticals}

Multiple non-natural chemicals/medications also exhibit antioxidant properties and are effective against VC. We identified 19 types of such candidates with potential therapeutic effects against VC: acetylcysteine ${ }^{33}$, alendronate ${ }^{49}$, BMP inhibitor ${ }^{39}$, dimethyl fumarate ${ }^{47}$, farnesyltransferase inhibitor $^{41}$, gemigliptin ${ }^{57}$, growth hormone-releasing hormone (GHRH) receptor agonist ${ }^{58}$, metformin ${ }^{42}$, pentoxifylline $^{64}$, pravastatin ${ }^{33}$, pyrrolidine dithiocarbamate $(\text { PDTC })^{34}$, simvastatin ${ }^{32}$, sodium hydrosulfide ${ }^{53}$, sodium selenite $^{37,45}$, STS $^{36,43,44}$, Tempol $^{40}$, TRAM- $34^{46}$, topical poly(1,8-octamethylene-citrate-co-cysteine) $(\mathrm{POCC})^{54}$, and hydrogen peroxide ${ }^{51}$. Among these, 11 (57.9\%) were medications with specific clinical indications, while 8 (42.1\%) were non-medicinal chemicals. Seven of them (36.8\%) have ROS scavenging ability, while the other 12 (63.2\%) do not (Fig. 2). We have briefly summarized the action of each agent in the following section.

STS is first such chemical being tested for its activity against VC and reaps the most attention by researchers in this field. Originally used for the treatment of cyanide toxicity, STS is also found to have strong antioxidant properties and a calcium-chelating tendency ${ }^{79}$. Pasch et al. showed that STS significantly reduced VC severity in uremic rats, and they proposed that STS acted by inducing acidosis and urinary excretion of calcium $^{36}$. 


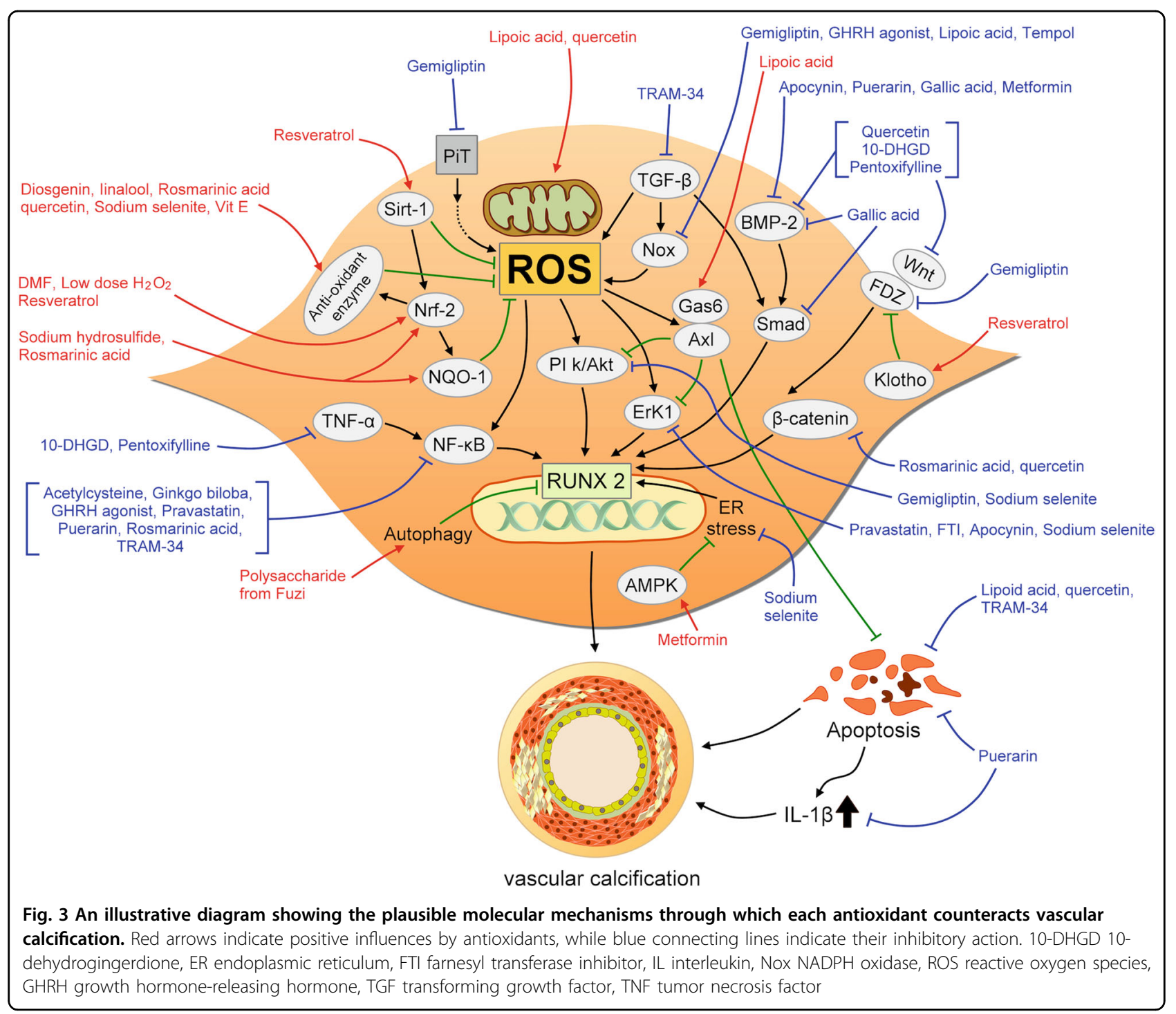

Subsequent studies suggested that STS could suppress BMP-2 expression and upregulate MGP in calcified $\mathrm{VSMCs}^{43}$, indicating that STS may interfere directly with the pathophysiology of $\mathrm{VC}$ in addition to its ability to bind calcium. With the assistance from adipocytes, STS may attenuate the calcification of arterial walls to a greater extent $^{44}$.

The most renowned antioxidant medication is probably acetylcysteine, which acts through its conversion into substrates for hepatic glutathione synthesis, and is used clinically for managing acetaminophen overdose and for prophylaxis of contrast-induced kidney injury ${ }^{80}$. While examining the vasculopathic effect of protease inhibitors, Afonso et al. found that acetylcysteine could retard the progression of VSMC calcification through its antioxidant and anti-inflammatory capability ${ }^{33}$. Sodium selenite, a formula that delivers the trace element selenium to host individuals, has been tested in two studies for its anti- calcification ability, since selenium restoration has been shown to raise glutathione peroxidase activity and to attenuate ROS production in patients with different illnesses ${ }^{81}$. Liu et al. revealed that sodium selenite could inhibit the ERK1/2 pathway, leading to a lower severity of calcification in a model of $\mathrm{H}_{2} \mathrm{O}_{2}$-induced VSMC phenotypic switch ${ }^{37}$. They also found that sodium selenite suppresses OS by elevating glutathione peroxidase activity, inhibiting $\mathrm{PI}_{3} \mathrm{~K} / \mathrm{Akt}$ activity and endoplasmic reticulum stress in $\mathrm{VSMCs}^{45}$, supporting its use as a treatment for VC.

Other medications, including anti-osteoporotic medication (alendronate), oral hypoglycemic agent (gemigliptin and metformin), hypolipidemic agents (pravastatin and simvastatin), medication for multiple sclerosis (dimethyl fumarate), microcirculatory insufficiency (pentoxifylline), and growth hormone deficiency (GHRH receptor agonist) have all been associated with suppressed 
ROS production and attenuated VC in different experimental models. Cutini et al. showed that alendronate increased NO production and activated MAPK signaling pathway, while it attenuated inflammation and reduced VC severity ${ }^{49}$. Ha et al. discovered that dimethyl fumarate administration led to Nrf2 activation, BMP-2 downregulation, and less severe VC in a calcified VSMC model and in animals with vitamin D-induced aortic calcification ${ }^{47}$. Pentoxifylline is also shown to decrease the expression of BMP-2, Wnt3a, and RANK in addition to its anti-inflammatory and anti-calcific properties ${ }^{64}$. Gemigliptin belongs to the dipeptidyl peptidase- 4 inhibitor class of antidiabetic agents, which exhibit a pleiotropic effect through glucagon-like peptide-1 (GLP-1)-dependent and -independent mechanisms including insulin sensitization and the inhibition of oxidation and apoptosis ${ }^{82}$. Choi et al. reported that gemigliptin attenuated VC through a variety of beneficial molecular alterations, including decreased expressions of PiT-1 and Nox4 with reduced $\mathrm{ROS}$ production, and suppressed $\mathrm{PI}_{3} \mathrm{~K}$-Akt and Wnt-FDZ pathway activities ${ }^{57}$. On the other hand, metformin has been shown to ameliorate VSMC calcification through AMPK-independent (BMP-2 inhibition) and AMPK-dependent (AMPK and eNOS activation) mechanisms ${ }^{42}$. Statins also exhibit anti-inflammatory and antioxidant properties, apart from their cholesterollowering effect. Ivanovski et al. first showed that simvastatin was capable of decreasing aortic OS markers and reducing aortic calcification in ApoE knockout mice with CKD, independent of cholesterol levels ${ }^{32}$. This is further supplemented by Afonso et al.'s work, which suggested that pravastatin could decrease local OS by inhibiting farnesyl transferase in calcified VSMCs ${ }^{33}$. GHRH receptor agonists, a hormonal agent not previously investigated for its vascular action, was shown to alleviate aortic calcification via NF- $\mathrm{KB}$ downregulation and the reduction in inflammation ${ }^{58}$. It seems that many of these antioxidant medications exert their actions by modulating NF- $\mathrm{kB}$ expression and lowering the contribution of inflammation to $\mathrm{VC}$ pathogenesis, although some positively influence vascular health through multiple mechanisms (Fig. 1). Judging from their efficacy against VC, it is expected that part of this medication list may eventually gain momentum as an effective therapy for VC treatment.

Several non-medicinal chemicals with antioxidant property are also found to protect against $\mathrm{VC}$, serving as potential treatment candidates. It was expected that BMP inhibitors LDN-193189 would retard VC progression in LDL receptor knockout mice; pharmacological BMP inhibition additionally attenuated the production of ROS and reduced the severity of inflammation in vascular tissues, as shown by Derwall et al. ${ }^{39}$. The inhibition of protein prenylation, which markedly influences isoprenoid synthesis and mevalonate metabolism, using farnesyl transferase inhibitor R115777 has also been shown to reduce aortic tissue OS levels and ameliorate calcium deposition by inhibiting Ras-Raf signaling ${ }^{41}$. Interestingly, calcium-activated potassium channel blocker TRAM-34, which helps maintain VSMC membrane potential and affects intracellular $\mathrm{Ca}^{2+}$ signaling, was found to decrease VSMC calcification by inducing NO release, attenuating TGF- $\beta$ signaling, and inhibiting NF- $\mathrm{kB}$ activation with reduced apoptosis ${ }^{46}$. The administration of sodium hydrosulfide, which strengthens endogenous antioxidant hydrogen sulfide action, was similarly found to reduce VC severity by decreasing $\mathrm{H}_{2} \mathrm{O}_{2}$ production, inhibiting TNF- $\alpha$ expression, and activating Nrf2-NQO1 pathway ${ }^{53}$. Dithiocarbamate compounds, such as PDTC, have been reported to exhibit an even stronger antioxidant property than $\mathrm{N}$-acetylcysteine by inhibiting NF- $\mathrm{KB}$ activation ${ }^{83}$. Mody et al. showed that PDTC could substantially halt the process of calcification in vascular cells, although the mechanisms involved have not been explored ${ }^{34}$.

Synthetic compounds exhibiting antioxidant properties are also being used to fight VC. Poly(1,8-octamethylene citrate) (POC), an elastomer constructed as a biodegradable material, has been shown previously to decrease ROS production in neighboring tissues when coated onto vascular grafts ${ }^{84}$. Its derivative, POCC, when coated, was also found to attenuate local oxidized DNA and lipid damages, accompanied by a reduction in graft calcification $^{54}$.

The confirmed effector mechanisms of these medicinal and non-medicinal chemicals with antioxidant properties against VC are summarized in an integrative way in Fig. 3.

\section{Antioxidants with contradictory findings regarding VC}

Some of these non-medicinal compounds have shown contradictory findings in the pathogenesis of VC. For example, tempol, a frequently used experimental superoxide dismutase mimetic, has been controversial regarding its effect on the progress of VC. Yamada et al. showed that tempol could reverse the osteoblastic transdifferentiation of VSMCs and improve uremic VC in CKD rats through ROS reduction and Nox 4 inhibition ${ }^{40}$. However, Liberman et al. showed that in a rabbit model of atherosclerotic calcification, although tempol lowered aortic ROS levels, it paradoxically increased medial calcification and plaque burden $^{24}$. This can be attributed to the species-dependent or calcification model-dependent actions of tempol, but further studies are needed before arriving at a definite conclusion. In addition, hydrogen peroxide is traditionally deemed an endogenous OS mediator and used exogenously as a ROS inducer. Intriguingly, Zhang et al. showed that a very low concentration $(0.01 \mathrm{mM})$ of hydrogen peroxide could on the contrary reduce intracellular ROS 
Table 2 Clinical studies investigating the therapeutic effect of anti-oxidants in patients with clinical VC

\begin{tabular}{|c|c|c|c|c|c|}
\hline Medications & Sample size & doses & Disease type & Response & Reference \\
\hline Sodium thiosulfate & $22 \mathrm{HD}$ & $\begin{array}{l}25 \mathrm{~g} \text { thrice weekly IV for } \\
3 \text { months }\end{array}$ & $\begin{array}{l}\text { Carotid, coronary, and } \\
\text { aortic calcification }\end{array}$ & Mean annual VC changes did not increase & 13 \\
\hline Sodium thiosulfate & $6 \mathrm{HD}$ & $\begin{array}{l}10 \mathrm{~g} \text { thrice weekly in } \\
\text { dialysate for } 6 \text { months }\end{array}$ & Coronary calcification & $\begin{array}{l}66 \% \text { had calcification reduction vs. } 0 \% \text { in } \\
\text { control }\end{array}$ & 14 \\
\hline Sodium thiosulfate & 18 HD & $\begin{array}{l}10 \mathrm{~g} \text { thrice weekly IV for } \\
6 \text { months }\end{array}$ & $\begin{array}{l}\text { Abdominal aortic } \\
\text { calcification }\end{array}$ & $\begin{array}{l}\text { Modest calcification reduction but moderate } \\
\text { leg pain improvement }\end{array}$ & 15 \\
\hline Sodium thiosulfate & $38 \mathrm{HD}$ & $\begin{array}{l}0.18 \mathrm{~g} / \mathrm{kg} \text { thrice weekly IV } \\
\text { for } 3 \text { months }\end{array}$ & Coronary calcification & $\begin{array}{l}\text { Treatment group had stable calcification } \\
\text { while control group progressed }\end{array}$ & 16 \\
\hline Cerium nitrate & 71 & $\begin{array}{l}\text { Topical application } \\
\text { to wound }\end{array}$ & Calciphylaxis & OR 0.44 (0.2-0.99) for mortality & 17 \\
\hline
\end{tabular}

$H D$ hemodialysis, IV intravenous, OR odds ratio, VC vascular calcification

generation and stimulate Nrf2 activity, decreasing the severity of VSMC calcification in vitro without inducing apoptosis $^{51}$. This phenomenon is reminiscent of the physiological role of "preconditioning" maneuver, which protects specific organs from severe damage if they are subjected to mild insult beforehand. Nonetheless, their findings are still preliminary and need replication.

\section{Clinically tested antioxidants for treatment of VC}

We identified five clinical studies testing one parenteral and the other topical agent with an antioxidative ability regarding their efficacy in treating VC (Table 2$)^{13-17}$. STS, which has been repeatedly tested in experimental models for inhibiting VC progression, is also used clinically to treat calcification involving arteries from different anatomical locations. However, it is evident that these studies are anecdotal, enrolling few patients, and the results appear heterogeneous with some showing calcification regression $^{14,15}$ while others reporting no interval changes $^{13,16}$. A topical chemical, cerium nitrate, has been shown to improve the overall outcome of patients with calciphylaxis when used for wound dressing, presumably through ROS scavenging and chelating local calcium ${ }^{17}$. Further studies are still needed to confirm these findings.

Finally, we undertook a comprehensive search in the clinicaltrials.gov registry to look for any completed or ongoing clinical trials involving the use of these 36 antioxidative compounds for treating VC, up to 7 June 2019. Only two registered trials attempted to test these agents against the target phenotype, $\mathrm{VC}$, including alendronate and statin, although the results have not been available. It is anticipated that more antioxidative compounds will gain momentum as anti-VC medications in the future.

\section{Conclusion and future perspective}

Natural compounds are rich sources of potential therapeutic candidates for various diseases, including neurodegenerative disorders, metabolic dysregulation, cardiovascular diseases ${ }^{85}$, and VC. Repurposing the existing medications for alternative applications is an important way of identifying novel therapies with known safety profiles ${ }^{86}$. For VC, the pivotal components in its pathogenesis are ROS production and inflammation ${ }^{87,88}$, which are targeted by many natural compounds and existing medications. Moreover, foods rich in these natural compounds may also be beneficial for patients with $\mathrm{VC}$, although the ingestion amount needed to achieve the optimal result remains elusive. With the knowledge gained by the current literature, we expect that more antioxidants will emerge in the future as potential candidates in the therapeutic armamentarium against VC.

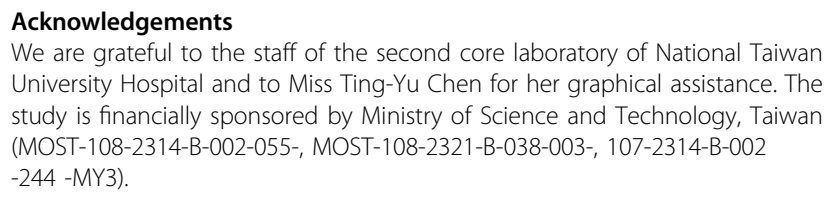

We are grateful to the staff of the second core laboratory of National Taiwan University Hospital and to Miss Ting-Yu Chen for her graphical assistance. The study is financially sponsored by Ministry of Science and Technology, Taiwan (MOST-108-2314-B-002-055-, MOST-108-2321-B-038-003-, 107-2314-B-002 $-244-M Y 3)$.

Consent for publication

Not applicable.

\begin{abstract}
Author details
${ }^{1}$ Department of Medicine, National Taiwan University Hospital BeiHu Branch, College of Medicine, National Taiwan University, Taipei, Taiwan. ${ }^{2}$ Department of Internal Medicine, National Taiwan University College of Medicine, Taipei, Taiwan. ${ }^{3}$ Graduate Institute of Toxicology, National Taiwan University College of Medicine, Taipei, Taiwan. ${ }^{4}$ School of Big Data Management, Soochow University, Taipei, Taiwan. ${ }^{5}$ Nephrology Division, Department of Internal Medicine, National Taiwan University Hospital, Taipei, Taiwan
\end{abstract}

Data availability

Literature summary data will be available upon reasonable request.

Conflict of interest

The authors declare that they have no conflict of interest.

Ethic approval and consent to participate

Not applicable. 


\section{Publisher's note}

Springer Nature remains neutral with regard to jurisdictional claims in

published maps and institutional affiliations.

Received: 26 August 2019 Revised: 23 September 2019 Accepted: 27 September 2019

Published online: 13 November 2019

\section{References}

1. Cherepanov, D. et al. Real-world cardiovascular disease burden in patients with atherosclerotic cardiovascular disease: a comprehensive systematic literature review. Curr. Med. Res. Opin. 34, 459-473 (2018).

2. Roth, G. A. et al. Demographic and epidemiologic drivers of global cardiovascular mortality. N. Engl. J. Med. 372, 1333-1341 (2015).

3. Covic, A. et al. Bone and mineral disorders in chronic kidney disease: implications for cardiovascular health and ageing in the general population. Lancet Diabetes Endocrinol. 6, 319-331 (2018).

4. Greif, M. et al. Lipoprotein (a) is independently correlated with coronary artery calcification. Eur. J. Intern. Med. 24, 75-79 (2013).

5. Malik, S. et al. Coronary artery calcium score for long-term risk classification in individuals with type 2 diabetes and metabolic syndrome from the multiethnic study of atherosclerosis. JAMA Cardiol. 2, 1332-1340 (2017).

6. Nitta, K., Ogawa, T., Hanafusa, N. \& Tsuchiya, K. Recent advances in the management of vascular calcification in patients with end-stage renal disease. Contrib. Nephrol. 198, 62-72 (2019).

7. Leopold, J. A. Vascular calcification: mechanisms of vascular smooth muscle cell calcification. Trends Cardiovasc. Med. 25, 264-274 (2015).

8. Shanahan, C. M., Crouthamel, M. H., Kapustin, A. \& Giachelli, C. M. Arterial calcification in chronic kidney disease: key roles for calcium and phosphate. Circ. Res. 109, 697-711 (2011).

9. Ho, C. Y. \& Shanahan, C. M. Medial arterial calcification. Arterioscler. Thromb. Vasc. Biol. 36, 1475-1482 (2016).

10. Byon, $\mathrm{C} . \mathrm{H}$. et al. Oxidative stress induces vascular calcification through modulation of the osteogenic transcription factor Runx2 by AKT signaling. J. Biol. Chem. 283, 15319-15327 (2008).

11. Durik, M. et al. Nucleotide excision DNA repair is associated with age-related vascular dysfunction. Circulation 126, 468-478 (2012).

12. Al-Aly, Z. Phosphate, oxidative stress, and nuclear factor-kB activation in vascular calcification. Kidney Int. 79, 1044-1047 (2011).

13. Mathews, S. J. et al. Effects of sodium thiosulfate on vascular calcification in end-stage renal disease: a pilot study of feasibility, safety and efficacy. Am. J. Nephrol. 33, 131-138 (2011).

14. Yonova, D. H. et al. First impressions of cardiovascular calcification treatment in hemodialysis patients with a new dialysis fluid containing sodium thiosulphate (STS). Int. J. Artif. Organs 37, 308-314 (2014).

15. Ghiandai, G., Ralli, C., Imperiali, P., Zingarelli, A. \& Duranti, E. Is the sodium thiosulfate therapy useful for vascular calcification in dialysis Pts? G. Ital. Nefrol. 32, gin/32.3.6 (2015)

16. Yu, Y., Bi, Z. M., Wang, Y., Chen, Z. Q. \& Xu, S. W. Effect of sodium thiosulfate on coronary artery calcification in maintenance hemodialysis patients. Zhonghua Yi Xue Za Zhi 96, 3724-3748 (2016).

17. Darres, A. et al. The effectiveness of topical cerium nitrate-silver sulfadiazine application on overall outcome in patients with calciphylaxis. Dermatology 235, 120-129 (2019).

18. Brodeur, M. R. et al. Reduction of advanced-glycation end products levels and inhibition of RAGE signaling decreases rat vascular calcification induced by diabetes. PLOS ONE 9, e85922 (2014).

19. Feng, W. et al. Apocynin attenuates angiotensin II-induced vascular smooth muscle cells osteogenic switching via suppressing extracellular signalregulated kinase 1/2. Oncotarget 7, 83588-83600 (2016).

20. Manivannan, J. et al. Diosgenin attenuates vascular calcification in chronic renal failure rats. Mol. Cell. Biochem. 378, 9-18 (2013).

21. Manivannan, J., Shanthakumar, J., Arunagiri, P., Raja, B. \& Balamurugan, E. Diosgenin interferes coronary vasoconstriction and inhibits osteochondrogenic transdifferentiation of aortic VSMC in CRF rats. Biochimie 102, 183-187 (2014).

22. Liberman, M. et al. Oxidant generation predominates around calcifying foci and enhances progression of aortic valve calcification. Arterioscler. Thromb. Vasc. Biol. 28, 463-470 (2008).
23. Kim, $\mathrm{H}$. et al. a-Lipoic acid attenuates vascular calcification via reversal of mitochondrial function and restoration of Gas6/Axl/Akt survival pathway. J. Cell. Mol. Med. 16, 273-286 (2012).

24. Bassi, E., Liberman, M., Martinatti, M. K., Bortolotto, L. A. \& Laurindo, F. R. M. Lipoic acid, but not tempol, preserves vascular compliance and decreases medial calcification in a model of elastocalcinosis. Braz. J. Med. Biol. Res. 47, 119-127 (2014)

25. Lu, T. et al. Induction of intracellular heat-shock protein 72 prevents the development of vascular smooth muscle cell calcification. Cardiovasc. Res. 96, 524-532 (2012).

26. Beazley, K. E. et al. Transglutaminase inhibitors attenuate vascular calcification in a preclinical model. Arterioscler. Thromb. Vasc. Biol. 33, 43-51 (2013).

27. Beazley, K. E., Eghtesad, S. \& Nurminskaya, M. V. Quercetin attenuates warfarininduced vascular calcification in vitro independently from matrix Gla protein. J. Biol. Chem. 288, 2632-2640 (2013).

28. Beazley, K. E., Lima, F., Borras, T. \& Nurminskaya, M. Attenuation of chondrogenic transformation in vascular smooth muscle by dietary quercetin in the MGP-deficient mouse model. PLOS ONE 8, e76210 (2013).

29. Chang, X. et al. Quercetin attenuates vascular calcification through suppressed oxidative stress in adenine-induced chronic renal failure rats. Biomed. Res. Int. 2017, 5716204 (2017).

30. Cui, L., Li, Z., Chang, X., Cong, G. \& Hao, L. Quercetin attenuates vascular calcification by inhibiting oxidative stress and mitochondrial fission. Vasc. Pharmacol. 88, 21-29 (2017).

31. Liang, Q., Chen, Y., Li, C. \& Lu, L. Quercetin attenuates Ox-LDL-induced calcification in vascular smooth muscle cells by regulating ROS-TLR4 signaling pathway. Nan Fang. Yi Ke Da Xue Xue Bao 38, 980-985 (2018).

32. Ivanovski, O. et al. Effect of simvastatin in apolipoprotein $\mathrm{E}$ deficient mice with surgically induced chronic renal failure. J. Urol. 179, 1631-1636 (2008).

33. Afonso, P. et al. LMNA mutations resulting in lipodystrophy and HIV protease inhibitors trigger vascular smooth muscle cell senescence and calcification: role of ZMPSTE24 downregulation. Atherosclerosis 245, 200-211 (2016).

34. Mody, N., Parhami, F., Sarafian, T. A. \& Demer, L. L. Oxidative stress modulates osteoblastic differentiation of vascular and bone cells. Free Radic. Biol. Med. 31, 509-519 (2001).

35. Peralta-Ramírez, A. et al. Vitamin E protection of obesity-enhanced vascular calcification in uremic rats. Am. J. Physiol. Ren. Physiol. 306, F422-F429 (2013).

36. Pasch, A. et al. Sodium thiosulfate prevents vascular calcifications in uremic rats. Kidney Int. 74, 1444-1453 (2008).

37. Liu, H., Lu, Q. \& Huang, K. Selenium suppressed hydrogen peroxide-induced vascular smooth muscle cells calcification through inhibiting oxidative stress and ERK activation. J. Cell. Biochem. 111, 1556-1564 (2010).

38. Roman-Garcia, P., Barrio-Vazquez, S., Fernandez-Martin, J. L., Ruiz-Torres, M. P. \& Cannata-Andia, J. B. Natural antioxidants and vascular calcification: a possible benefit. J. Nephrol. 24, 669-672 (2011).

39. Derwall, M. et al. Inhibition of bone morphogenetic protein signaling reduces vascular calcification and atherosclerosis. Arterioscler. Thromb. Vasc. Biol. 32, 613-622 (2012).

40. Yamada, S. et al. The antioxidant tempol ameliorates arterial medial calcification in uremic rats: Important role of oxidative stress in the pathogenesis of vascular calcification in chronic kidney disease. J. Bone Miner. Res. 27, 474-485 (2012).

41. Nikolov, I. G. et al. Farnesyltransferase inhibitor R115777 protects against vascular disease in uremic mice. Atherosclerosis 229, 42-51 (2013).

42. Cao, X. et al. Metformin inhibits vascular calcification in female rat aortic smooth muscle cells via the AMPK-eNOS-NO pathway. Endocrinology 154, 3680-3689 (2013).

43. Zhong, H., Liu, F., Dai, X., Zhou, L. \& Fu, P. Sodium thiosulfate protects human aortic smooth muscle cells from osteoblastic transdifferentiation via high-level phosphate. Kaohsiung J. Med. Sci. 29, 587-593 (2013).

44. Chen, N. X., O'Neill, K., Akl, N. K. \& Moe, S. M. Adipocyte induced arterial calcification is prevented with sodium thiosulfate. Biochem. Biophys. Res. Commun. 449, 151-156 (2014).

45. Liu, H., Li, X., Qin, F. \& Huang, K. Selenium suppresses oxidative-stressenhanced vascular smooth muscle cell calcification by inhibiting the activation of the PI3KJAKT and ERK signaling pathways and endoplasmic reticulum stress. J. Biol. Inorg. Chem. 19, 375-388 (2014).

46. Freise, C. \& Querfeld, U. Inhibition of vascular calcification by block of intermediate conductance calcium-activated potassium channels with TRAM-34. Pharmacol. Res. 85, 6-14 (2014).

47. Ha, C.-M. et al. Activation of Nrf2 by dimethyl fumarate improves vascular calcification. Vasc. Pharmacol. 63, 29-36 (2014). 
48. Kee, H. J. et al. Gallic acid inhibits vascular calcification through the blockade of BMP2-Smad1/5/8 signaling pathway. Vasc. Pharmacol. 63 71-78 (2014)

49. Cutini, P. H., Rauschemberger, M. B., Sandoval, M. J. \& Massheimer, V. L. Vascular action of bisphosphonates: in vitro effect of alendronate on the regulation of cellular events involved in vessel pathogenesis. J. Mol. Cell. Cardiol. 100, 83-92 (2016).

50. Li, E.-G., Tian, J. \& Xu, Z.-H. Effects of Gingko biloba extract (EGb 761) on vascular smooth muscle cell calcification induced by $\beta$-glycerophosphate. Ren. Fail. 38, 552-557 (2016).

51. Zhang, W., Li, Y., Ding, H., Du, Y. \& Wang, L. Hydrogen peroxide prevents vascular calcification induced ROS production by regulating Nrf-2 pathway. Ren. Fail. 38, 1099-1106 (2016).

52. Zhang, $\mathrm{P}$. et al. Resveratrol ameliorated vascular calcification by regulating Sirt-1 and Nrf2. Transplant. Proc. 48, 3378-3386 (2016).

53. Aghagolzadeh, P. et al. Hydrogen sulfide attenuates calcification of vascular smooth muscle cells via KEAP1/NRF2/NQO1 activation. Atherosclerosis 265, 78-86 (2017).

54. Jiang, B. et al. Vascular scaffolds with enhanced antioxidant activity inhibit graft calcification. Biomaterials 144, 166-175 (2017).

55. Rios, R. et al. Vitamin E protects against extraskeletal calcification in uremic rats fed high fat diets. BMC Nephrol. 18, 374 (2017).

56. Jordão, J. B., Porto, H. K., Lopes, F. M., Batista, A. C. \& Rocha, M. L. Protective effects of ellagic acid on cardiovascular injuries caused by hypertension in rats. Planta Med. 83, 830-836 (2017).

57. Choi, S.-Y. et al. Dipeptidyl peptidase-4 inhibitor gemigliptin protects against vascular calcification in an experimental chronic kidney disease and vascular smooth muscle cells. PLOS ONE 12, e0180393 (2017).

58. Shen, J. et al. Regulation of vascular calcification by growth hormone-releasing hormone and its agonists. Circ. Res. 122, 1395-1408 (2018).

59. Salimi, F., Jafari-Nodooshan, S., Zohourian, N., Kolivand, S. \& Hamedi, J. Simultaneous anti-diabetic and anti-vascular calcification activity of Nocardia sp. UTMC 751. Lett. Appl. Microbiol. 66, 110-117 (2018).

60. Liao, L. et al. Polysaccharide from Fuzi protects against Ox-LDL-induced calcification of human vascular smooth muscle cells by increasing autophagic activity. Mol. Med. Rep. 17, 5109-5115 (2018).

61. Kaur, T., Kaul, S. \& Bhardwaj, A. Efficacy of linalool to ameliorate uremia induced vascular calcification in wistar rats. Phytomedicine 51, 191-195 (2018).

62. Liu, H. et al. Puerarin inhibits vascular calcification of uremic rats. Eur. J. Pharmacol. 855, 235-243 (2019).

63. Ji, R. et al. Rosmarinic acid exerts an antagonistic effect on vascular calcification by regulating the Nrf2 signalling pathway. Free Radic. Res. 53, 187-197 (2019).

64. Elseweidy, M. M. et al. Potential therapeutic roles of 10-dehydrogingerdione and/or pentoxifylline against calcium deposition in aortic tissues of high dietary cholesterol-fed rabbits. Mol. Cell. Biochem. 453, 131-142 (2019).

65. Simonyi, A. et al. The neuroprotective effects of apocynin. Front. Biosci. 4, 2183-2193 (2012)

66. Stefanska, J. \& Pawliczak, R. Apocynin: molecular aptitudes. Mediators Inflamm. 2008, 106507 (2008).

67. Sethi, G. et al. Pro-apoptotic and anti-cancer properties of diosgenin: a comprehensive and critical review. Nutrients 10,645 (2018).

68. Andres, S. et al. Safety aspects of the use of quercetin as a dietary supplement. Mol. Nutr. Food Res. 62, 1700447 (2018).
69. Choi, S.-Y., Park, G.-S., Lee, S.-Y., Kim, J.-Y. \& Kim, Y.-K. The conformation and CETP inhibitory activity of [10]-dehydrogingerdione isolated from Zingiber officinale. Arch. Pharm. Res. 34, 727-731 (2011).

70. Elseweidy, M. M., Abdallah, F. R., Younis, N. N., Aldohmy, S. \& Kassem, H. M. 10 Dehydrogingerdione raises $\mathrm{HDL}$-cholesterol through a CETP inhibition and wards off oxidation and inflammation in dyslipidemic rabbits. Atherosclerosis 231, 334-340 (2013).

71. Mirzaei, $H$. et al. Phytosomal curcumin: a review of pharmacokinetic, experimental and clinical studies. Biomed. Pharmacother. 85, 102-112 (2017).

72. Federico, A., Dallio, M. \& Loguercio, C. Silymarin/silybin and chronic liver disease: a marriage of many years. Molecules 22, 191 (2017).

73. Ceci, $\mathrm{C}$. et al. Experimental evidence of the antitumor, antimetastatic and antiangiogenic activity of ellagic acid. Nutrients 10, 1756 (2018).

74. Pietri, S., Maurelli, E., Drieu, K. \& Culcasi, M. Cardioprotective and anti-oxidant effects of the terpenoid constituents of ginkgo biloba extract (EGb 761). J. Mol. Cell. Cardiol. 29, 733-742 (1997).

75. Liu, B., Tan, Y., Wang, D. \& Liu, M. Puerarin for acute ischaemic stroke. Cochrane Database Syst. Rev. 2, CD004955 (2016).

76. Baur, J. A. \& Sinclair, D. A. Therapeutic potential of resveratrol: the in vivo evidence. Nat. Rev. Drug Discov. 5, 493-506 (2006).

77. Lapczynski, A., Letizia, C. S. \& Api, A. M. Fragrance material review on d-linalool. Food Chem. Toxicol. 46, S193-S194 (2008).

78. Elufioye, T. O. \& Habtemariam, S. Hepatoprotective effects of rosmarinic acid: Insight into its mechanisms of action. Biomed. Pharmacother. 112, 108600 (2019).

79. Pouyatos, B., Gearhart, C., Nelson-Miller, A., Fulton, S. \& Fechter, L. Oxidative stress pathways in the potentiation of noise-induced hearing loss by acrylonitrile. Hear. Res. 224, 61-74 (2007).

80. Rushworth, G. F. \& Megson, I. L. Existing and potential therapeutic uses for Nacetylcysteine: the need for conversion to intracellular glutathione for antioxidant benefits. Pharmacol. Ther. 141, 150-159 (2014).

81. Manzanares, W. et al. High-dose selenium reduces ventilator-associated pneumonia and illness severity in critically ill patients with systemic inflammation. Intensive Care Med. 37, 1120-1127 (2011).

82. Aroor, A. R., Sowers, J. R., Jia, G. \& DeMarco, V. G. Pleiotropic effects of the dipeptidylpeptidase-4 inhibitors on the cardiovascular system. Am. J. Physiol. Heart Circ. Physiol. 307, H477-H492 (2014).

83. Zhu, B.-Z., Carr, A. C. \& Frei, B. Pyrrolidine dithiocarbamate is a potent antioxidant against hypochlorous acid-induced protein damage. FEBS Lett. 532 80-84 (2002).

84. van Lith, R., Gregory, E. K., Yang, J., Kibbe, M. R. \& Ameer, G. A. Engineering biodegradable polyester elastomers with antioxidant properties to attenuate oxidative stress in tissues. Biomaterials 35, 8113-8122 (2014).

85. Kunal, S., Jawed, A. S., Gaurav, S., Rakesh, M. \& Naibedya, C. Role of phytochemicals in the prevention of menopausal bone loss: evidence from in vitro and in vivo, human interventional and pharmacokinetic studies. Curr. Med. Chem. 16, 1138-1157 (2009).

86. Cha, Y. et al. Drug repurposing from the perspective of pharmaceutical companies. Br. J. Pharmacol. 175, 168-180 (2018).

87. Chao, C.-T. et al. Circulating microRNA-125b predicts the presence and progression of uremic vascular calcification. Arterioscler. Thromb. Vasc. Biol. 37 1402-1414 (2017)

88. Chao, C.-T, Yeh, H.-Y., Yuan, T.-H., Chiang, C.-K. \& Chen, H.-W. MicroRNA-125b in vascular diseases: an updated systematic review of pathogenetic implications and clinical applications. J. Cell. Mol. Med. 23, 5884-5894 (2019). 